\title{
Effect of extended-term estrogen on voiding in a postpartum ovariectomized rat model
}

\author{
Narihiko Hayashi, MD; Anthony J. Bella, MD; Guifang Wang, MD; Guiting Lin, MD, PhD; Donna Y. Deng, MD; \\ Lora Nunes; Tom F. Lue, MD
}

\section{ABSTRACT}

Introduction: We tested the hypothesis that extended-term (5-week) estrogen therapy would negatively impact voiding function in a postpartum, ovariectomized rat model.

\begin{abstract}
Methods: Immediately after delivery, 30 primiparous Sprague-Dawley rats underwent intravaginal balloon dilation, followed by ovariectomy 1 week later. Cystometry at postpartum week 2 determined normal or abnormal voiding patterns. After randomization, one-half the normal and abnormal voiding rats received 5 weeks of estrogen therapy, while the remainder received placebo. Estrogen effect was determined by repeat cystometry and immunohistochemical analysis of the urethra and vagina.
\end{abstract}

Results: Abnormal voiding increased from $60.0 \%$ to $73.3 \%$ in the estrogentreated group and declined from $60 \%$ to $33 \%$ for the placebo group. Rats were then divided into 4 groups for comparison: normal voiding versus placebo (group 1), abnormal voiding versus placebo (group 2), normal voiding versus estrogen (group 3) and abnormal voiding versus estrogen (group 4). Bladder capacity, leak point pressure and maximum voiding pressure were most depressed in group 4. Estrogen treatment was associated with a significant downregulation of $\alpha_{1 \mathrm{~A}}$ and $\alpha_{1 \mathrm{D}}$-adrenoceptors in the urethral submucosa but an upregulation of $\mathrm{nNOS}$ in the urethral smooth muscle.

Conclusion: Extended-term estrogen therapy in a rat model of simulated birth trauma and ovariectomy resulted in a higher rate of incontinence. Immunohistochemical examination demonstrated significant downregulation of urethral $\alpha_{1 \mathrm{~A}}$-and $\alpha_{1 \mathrm{D}}$-adrenoceptors and upregulation of neuronal nitric oxide synthase (nNOS) in the urethra of estrogen-treated groups. These studies question the use of hormone replacement therapy in the treatment of postmenopausal incontinence.

CUAJ 2007;1(3):256-63

\section{Introduction}

After detrusor overactivity, stress urinary incontinence (SUI) is the second most common type of urinary leakage in the elderly. SUI in women has an overall prevalence of approximately $10 \%-30 \%$ between the ages of 15 and 64 years and results in significant patient morbidity and billions of dollars in health care expenditures each year. ${ }^{1} \mathrm{SUI}$ is defined as the involuntary loss of urine through the urethral meatus when bladder pressure exceeds urethral pressure in the absence of detrusor activity; risk factors include pregnancy, childbirth, parity, hysterectomy, menopause, obesity, diabetes, chronic respiratory disease and conditions related to impaired pelvic floor innervation. ${ }^{2}$

Hormonal changes linked with aging may also contribute to the development of urinary disorders. An increase in urinary symptoms, including incontinence, frequency, urgency and recurrent infection, has been reported after menopause. ${ }^{3}$ Several small scale clinical trials support the commonly held notion that exogenous estrogen may improve urinary function and decrease incontinence rates. ${ }^{4-6}$ Hormone replacement therapy (HRT) has thus prevailed as a leading clinical treatment to reverse genital atrophy and improve urethral closing pressure.

Recently, long-term studies involving thousands of women indicated that hormonal therapy neither improves continence in women with existing urinary incontinence nor prevents the development of incontinence in continent individuals. In the Heart and Estrogen-Progestin Replacement Study (HERS), a randomized, blinded study of 2763 postmenopausal women under age 80 years with coronary disease and intact uteri, the physiological effects of HRT were evaluated. Participants were randomly assigned to $0.625 \mathrm{mg}$ of conjugated estrogens plus $2.5 \mathrm{mg}$ of medroxyprogesterone acetate in 1 daily tablet or placebo and were observed for a mean of 4.1 years. Incontinence improved in $26 \%$ of the women assigned to placebo versus $21 \%$ assigned to the hormone group, while only $27 \%$ of the placebo group worsened, compared with $39 \%$ of the hormone group $(p=0.001)$. Based on significant findings evident by 4 months of therapy, the authors concluded that HRT is not recommended for the treatment of incontinence. ${ }^{7}$ Additionally, during clinical testing of levormeloxifene, a selective estrogen receptor modulator (SERM), 
women who received the drug had a marked increase in urinary incontinence, compared with those who took placebo $(17 \%$ v. $4 \%)$ as well as an increase in voiding frequency, endometrial thickness and uterine enlargement. Secondary to the number and severity of adverse events, the trial was prematurely halted after 10 months. ${ }^{8}$

Over the past 7 years, our laboratory has investigated the influence of simulated birth trauma and hormonal deficiency on urinary continence in a postpartum, ovariectomized female rat model. . $^{9-11}$ We determined that prolonged labour and ovariectomy induced higher rates of SUI versus ovariectomy alone. Further, our results confirmed that muscular and neurological alterations in the urethra and pelvic floor likely play a role in the development of SUI. ${ }^{11}$

Although scientific data are available regarding the effects of female hormones on the vagina, uterus and bladder, examination of the urethra has largely been neglected to date. The objectives of this study were to evaluate the effect of estrogen replacement on voiding function in our postpartum, ovariectomized rat model and identify the immunohistochemical alterations of the urethra and vagina.

\section{Materials and Methods}

\section{Experimental design}

This study was approved by the local ethical committee for experimentation (University of California, San Francisco, Institutional and Animal Care Use Committee) and complied with National Institutes of Health $(\mathrm{NIH})$ regulations for the care and use of laboratory animals. Thirty primiparous pregnant 2-month-old Sprague-Dawley female rats at gestational day 16 (230-280 g) were housed at a constant $16^{\circ} \mathrm{C}$ room temperature and $47 \%$ humidity, with a 12-hour light-dark cycle. Immediately postpartum, animals underwent intravaginal balloon dilation under anesthesia and 1 week later underwent bilateral ovariectomy (Fig. 1). Two weeks postpartum, initial transvesical cystometry was performed. Based on the urodynamic findings, each rat was classified as having normal (NV) or abnormal voiding (AV). After cystometry, AV animals outnumbered the NV group (18:12), and we felt that the comparison would be difficult after estrogen treatment. Therefore, the 2 groups (NV v. AV) were randomly divided in half and were cross-mixed, establishing 2 equal groups of 15 animals each. At the 3 -week mark, 1 group underwent dorsal subcutaneous implantation of a 0.5-mg $17 \beta$-estradiol pellet, while the other group received placebo. Estrogen or placebo pellet placement was repeated at 6 weeks to maintain constant drug bioavailability. Repeat cystometric results were obtained 7.5 weeks after balloon dilation, and the rats were again evaluated with urodynamic studies. For final outcome analyses, animals were grouped as follows: 1 ) NV plus placebo; 2) AV plus placebo; 3) NV plus estrogen; 4) AV plus estrogen (Fig. 1). At sacrifice, the pelvic tissues, midurethra and vagina were harvested. The midurethra was chosen because it represents the thickest region of the urethra with both striated and smooth muscle components and has demonstrated the least anatomic variation in our previous studies.

\section{Intravaginal balloon inflation}

Immediately postpartum, all rats underwent anesthesia using intraperitoneal injections of ketamine $(90 \mathrm{mg} / \mathrm{kg})$ and xylazine $(10 \mathrm{mg} / \mathrm{kg})$. To simulate prolonged labour, a modified distal-tip shortened $22 \mathrm{Fr}$ Foley catheter was inserted transvaginally and inflated (3 mL sterile water). A 130-g weight was attached to the catheter's proximal end. The rat was placed in a fixed prone position at the edge of the mini operating table, leaving the caudal end of the animal hanging downward without touching the table. This allowed the weighted catheter to hang freely against gravity. The rat's colour, warmth and respirations were monitored without positional disturbance for 3 hours. This mechanism simulates the pressure effects of a large fetus on the pelvic floor during the second stage of labour and has been previously shown to create a consistent model of SUI. ${ }^{11}$ We used postpartum instead of virgin rats because it is more representative of the clinical situation for which most SUI occurs (after vaginal birth).

\section{Bilateral ovariectomy}

One week after balloon dilation, the rats were anesthetized as above. Using a small vertical lower 
Hayashi et al

midline abdominal incision, vascular pedicles were suture ligated and ovaries removed, followed by a 2-layer abdominal closure. We performed ovariectomy 1 week after vaginal dilation because performing both procedures at the same time increased the morbidity and mortality of the rats in our previous experiments.

\section{Transvesical cystometry}

Two weeks after delivery, under sedation with ketamine $(100 \mathrm{mg} / \mathrm{kg})$, a repeat lower midline incision exposed the bladder. A 27-gauge butterfly needle was inserted into the bladder dome, and all urine was evacuated. Using a 3-way stopcock, the

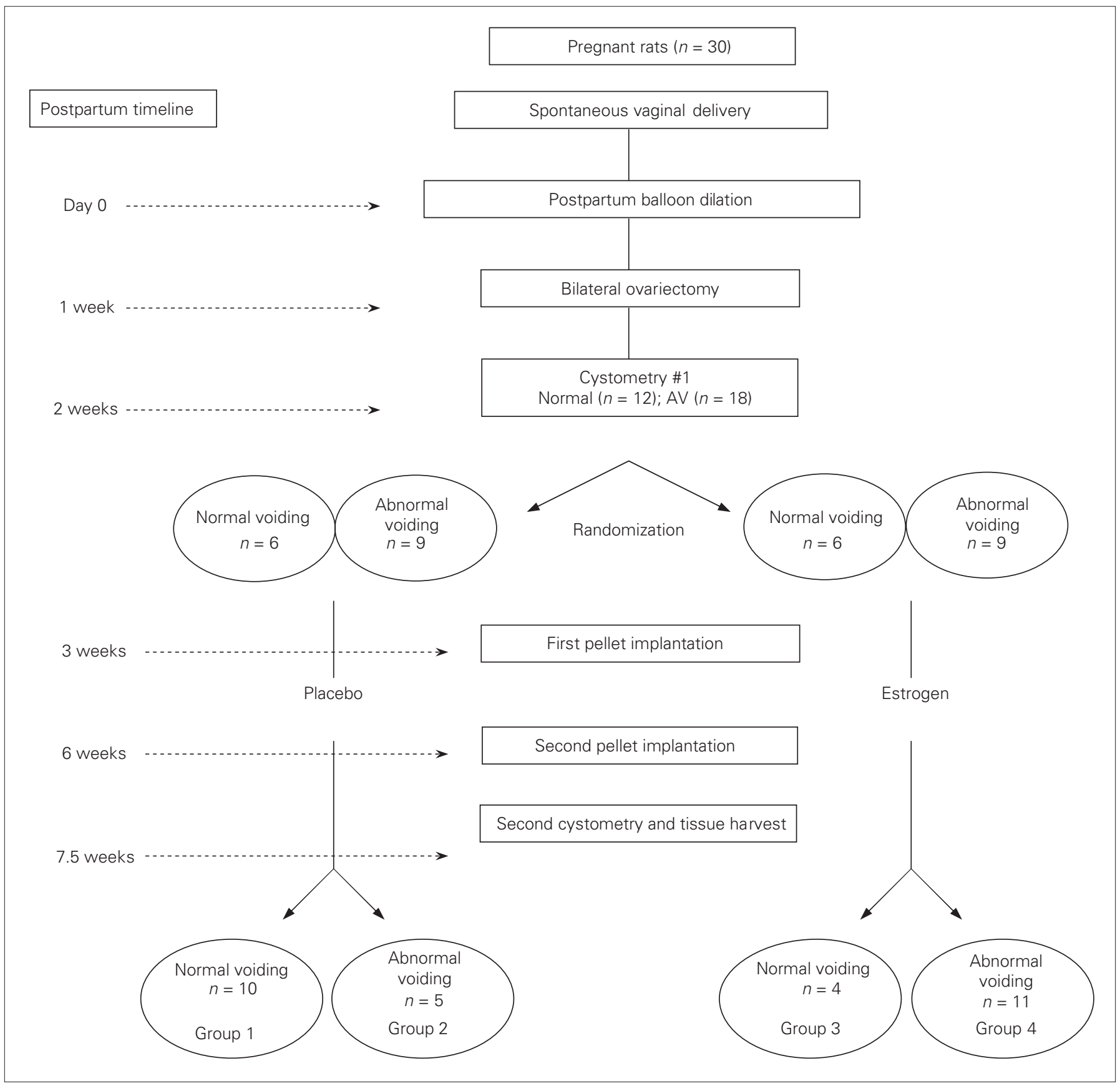

Fig. 1. Experimental design. $A V=$ abnormal voiding . 
needle was connected to both a Baxter Uniflow pressure transducer (Baxter Healthcare Corp., Irvine, Calif.) and a Harvard Model 22 infusion pump (Harvard, Millis, Mass.). Saline at $37^{\circ} \mathrm{C}$ was infused at $0.1 \mathrm{~mL}$ per minute after calibrating the pressure transducer to zero atmospheres. A customized Macintosh Quadra 800 (Apple Computer Inc., Cupertino, Calif.) with LabView 4.0 software (National Instruments Corp., Austin, Tex.) collected the data.

Voiding was considered normal if a detrusor contraction was followed by expulsion of saline from the meatus and with bladder emptying. The modified leak point pressure was determined at this point, and the instilled intravesical volume represented bladder capacity. A pattern of moderate incontinence was identified on cystometry if intermittent small leakage occurred with or without bladder contraction during filling, followed by a sustained detrusor contraction and voiding. Cystometric studies in animals with severe incontinence displayed a continued small amount of saline leakage at low detrusor pressures, without the bladder filling to capacity (Fig. 2). Urodynamics were repeated at 7.5 weeks postpartum. Because these studies were performed under ketamine sedation, we could not reliably differentiate overactive bladder from sphincter deficiency, as in the case of awake cystometry and therefore grouped them together as the AV group.

\section{Estrogen or placebo pellet implantation}

One week after initial cystometry and randomization, a 0.5 -mg $17 \beta$-estradiol $(21 \mathrm{~d}$ timed-release formula) or placebo pellet (Innovative Research of America, Sarasota, Fla.) was implanted in a subcutaneous pouch. After ketamine sedation, a 5-mm incision in the dorsal skin at shoulder height allowed pellet deposition. This procedure was repeated in all groups 3 weeks later to maintain constant estrogen or placebo levels. In our pilot studies, we did not notice any changes with shorter-term estrogen therapy, thus we implanted a second pellet at 3 weeks, when the first pellet's effects had terminated.

Upon completing the second cystometry at 7.5 weeks after delivery, animals were euthanized. The pelvic floor was exposed, and samples of the midurethra and vagina were removed.

\section{Immunohistochemistry and image analysis}

Tissue specimens were fixed in cold $2 \%$ formaldehyde and $0.002 \%$ saturated picric acid in $0.1 \mu \mathrm{mol} / \mathrm{L}$ phosphate buffer ( $\mathrm{pH}$ 8.0) for 4 hours, followed by overnight immersion in a buffer containing 30\% sucrose for cryoprotection. The specimens were embedded in OCT Compound (Sakura Finetic USA, Torrance, Calif.) and stored at $-70^{\circ} \mathrm{C}$ until use. Fixed frozen tissue specimens were cut at $5 \mu \mathrm{m}$ thickness, mounted to SuperFrost Plus charged slides (Fisher Scientific, Pittsburgh, Pa.) and air-dried for 5 minutes. Endogenous peroxidase activity was blocked with $0.3 \% \quad \mathrm{H}_{2} \mathrm{O}_{2}$

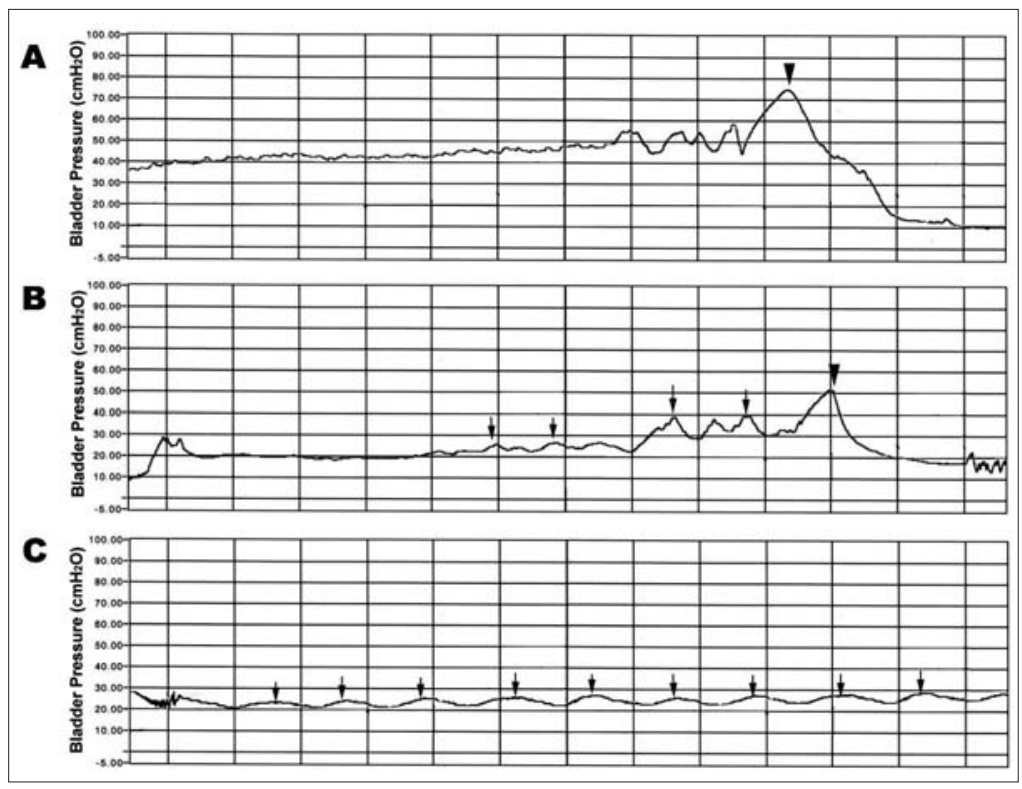

Fig. 2. Normal cystometry curve. Saline voiding from the urethra only occurs when the bladder contracts at capacity (large arrow) in response to filling (A). Moderate abnormal voiding. Small amount of saline leakage occur several times during filling (small arrows). Larger amounts of saline were voided at bladder capacity (large arrow) (B). Severe abnormal voiding. Saline continues to leak from the urethra (small arrows), inhibiting bladder filling to capacity (C).

\begin{tabular}{|c|c|c|}
\hline \multirow[b]{2}{*}{ Variable } & \multicolumn{2}{|c|}{ Voiding, mean (SEM) } \\
\hline & $\begin{array}{l}\text { Abnormal } \\
(n=18)\end{array}$ & $\begin{array}{l}\text { Normal } \\
(n=12)\end{array}$ \\
\hline Bladder capacity, mL & $0.82(0.2)^{*}$ & $1.53(0.2)$ \\
\hline Maximum pressure, $\mathrm{cm}$ water & $48.82(1.6)$ & $46.08(2.6)$ \\
\hline Leak point pressure, $\mathrm{cm}$ water & $43.94(1.8)$ & NA \\
\hline
\end{tabular}


methanol for 10 minutes. After rinsing, sections were washed twice in phosphate-buffered salene (PBS) for 5 minutes and incubated with 3\% horse serum in PBS with $0.3 \%$ Triton X-100 (Rohm and Haas Co., Spring House, Pa.) for 30 minutes (room temperature) to eliminate nonspecific protein binding. After draining, the slides were incubated overnight at $4{ }^{\circ} \mathrm{C}$ with goat polyclonal antibodies to $\alpha_{1 A^{-}}$or $\alpha_{1 D^{-}}$adrenoceptors (Santa Cruz Biotechnology, Santa Cruz, Calif; 1:100) or mouse monoclonal antibody to neuronal nitric oxide synthase (nNOS) (BD Transduction Laboratories, San Jose, Calif; 1:500) to examine potential changes in contractile ( $\alpha$ adrenergic) and relaxant (nitric oxide [NO]) properties of the urethral sphincter. Slides incubated without antibodies in a similar fashion served as negative controls. After washing with buffer sections, slides were immunostained with the avidin-biotin-peroxydase method (Elite ABC, Vector Laboratories, Burlingame, Calif.), using diaminobenzidine as the chromagen, followed by hematoxylin counterstaining. For image analysis, 5 randomly selected fields per tissue, per animal, for each treatment group were photographed and recorded with a Retiga Qimage dig- ital camera and ACT-1 software (Nikon Instruments Inc., Melville, NY). Images were quantified with Image-Pro Plus software (Media Cybernetics, Silver Spring, Md.). The ratio of the $\alpha_{1 A^{-}}$or $\alpha_{1 D^{-}}$-adrenoceptor or nNOS stained pixels to all pixels of the whole picture (equal to $100 \%$ ) provided a percentage. Different team members performed the functional study and histological evaluation, and evaluations were blinded until after the final analyses.

All values are expressed as mean and standard error of the mean. We used the unpaired 2-tailed $t$ test for statistical analysis, using YSTAT 2002 (Igakutosho, Tokyo, Japan). For posttreatment continence status, we performed the chi-squared test. Comparisons were considered significant at $p<0.05$.

\section{Results}

\section{Urodynamics}

Initial transvesical cystometric profiles performed 2 weeks after postpartum intravaginal balloon dilation and 1 week after ovariectomy identified 18 rats

\begin{tabular}{|c|c|c|c|c|}
\hline \multirow[b]{2}{*}{ Variable } & \multicolumn{2}{|c|}{ Placebo, mean (SEM)* } & \multicolumn{2}{|c|}{ Estrogen, mean (SEM)* } \\
\hline & $\begin{array}{c}\text { Group } 1 \\
\text { (NV } n=6)\end{array}$ & $\begin{array}{c}\text { Group 2 } \\
(\mathrm{AV} n=9)\end{array}$ & $\begin{array}{c}\text { Group } 3 \\
(\mathrm{NV} n=6)\end{array}$ & $\begin{array}{l}\text { Group 4, } \\
(\mathrm{AV} n=9)\end{array}$ \\
\hline Resolution of AV, no. & NA & 4 & NA & 0 \\
\hline Development of AV, no. & 0 & NA & 2 & NA \\
\hline Bladder capacity, $\mathrm{mL} \dagger$ & $1.3(0.1)$ & $1.4(0.1)$ & $1.1(0.1)$ & $0.85(0.1)$ \\
\hline Maximum pressure, $\mathrm{cm}$ water $\ddagger \S$ & $45.8(0.9)$ & $54.2(0.5)$ & $48.0(2.1)$ & $41.8(0.7)$ \\
\hline Leak point pressure, cm water & $47.5(4.3)$ & $45.8(1.9)$ & $44.5(1.3)$ & $38.6(1.3)$ \\
\hline \multicolumn{5}{|c|}{$\begin{array}{l}\text { SEM }=\text { standard error of the mean; } \mathrm{NV}=\text { normal voiding; } \mathrm{AV}=\text { abnormal voiding; } \mathrm{NA}=\text { not applicable. } \\
\text { *Unless otherwise indicated. } \\
\dagger p<0.05 \text { for group } 3 \mathrm{v} \text {. group } 4 \text {, and groups } 1 \text { and } 2 \mathrm{v} \text {. group } 4 . \\
\neq p<0.05 \text { group } 3 \mathrm{v} \text { group } 4 . \\
\$ p<0.05 \text { for group } 1 \text { and group } 2 \mathrm{v} \text {. group } 2 \text { and group } 4 \text {. } \\
\uparrow p<0.05 \text { for group } 3 \mathrm{v} \text {. group } 4 \text {, and groups } 1 \text { and } 2 \mathrm{v} \text {. group } 4 .\end{array}$} \\
\hline
\end{tabular}

\begin{tabular}{|c|c|c|c|c|c|c|}
\hline \multirow[b]{2}{*}{ Urethral or vaginal region } & \multicolumn{3}{|c|}{ Estrogen, mean (SEM) } & \multicolumn{3}{|c|}{ Placebo, mean (SEM) } \\
\hline & $\alpha 1 \mathrm{~A}$ & $\alpha 1 D$ & $\mathrm{nNos}$ & $\alpha 1 \mathrm{~A}$ & $\alpha 1 D$ & $\mathrm{nNos}$ \\
\hline Urethral epithelium & ME & ME & $78.11(61.9)$ & $\mathrm{ME}$ & $\mathrm{ME}$ & $18.8(0.8$ \\
\hline Urethral submucosa & $2.72(0.5)^{*}$ & $1.85(0.3)^{*}$ & & & & \\
\hline Urethral smooth muscle & ME & ME & $0.65(0.1)$ & $5.10(0.7)$ & $2.77(0.4)$ & ME \\
\hline Vaginal submucosa & $7.61(0.9)$ & $14.20(1.9)$ & ME & $0.41(0.1)$ & $0.97(0.2)$ & $\mathrm{ME}$ \\
\hline
\end{tabular}


(60\%) with AV patterns and $12(40 \%)$ with normal voiding. Table 1 compares urodynamic findings. Repeat cystometry after estrogen treatment documented an increase in AV from $60 \%$ to $73.3 \%$ (from 9 to 11 rats), whereas the placebo group demonstrated a decline in AV from $60 \%$ to $33.3 \%$ (from 9 to 5 rats). The difference is statistically significant ( $\chi^{2}$ of 4.821 and $\left.p=0.028\right)$. In a subset analysis, estrogen-treated rats with abnormal voiding (group 4) more frequently displayed a higher severity of incontinence, compared with placebotreated rats with AV (group 2), with continuous leakage hampering the ability to reach bladder capacity. Other parameters are summarized in Table 2, with statistically significant lower bladder capacity, maximal bladder pressure and modified leak point pressure noted in group 4.

\section{Immunohistochemistry}

We observed thickened epithelium and subepithelium, as well as increased amounts of smooth muscle fibres in the muscularis, for the estrogentreated vaginal tissue, and we observed no general morphological differences for urethral specimens between estrogen- and placebo-treated groups.

Alpha adrenoceptors $1_{\mathrm{A}}$ and $1_{\mathrm{D}}$ were identified in both the urethra and the vagina. Estrogen therapy significantly downregulated $\alpha_{1 A^{-}}$and $\alpha_{1 D^{-}}$ adrenoceptor expression in the endothelium and subendothelial regions of the urethra while increasing levels of both receptors in the vagina (Table 3, Fig. 3 and Fig. 4).

$\mathrm{nNOS}$ staining was significantly elevated in the urethral smooth muscle and urothelium, as well as in the basal layer of the vaginal epithelium of rates treated wtih estrogen (Fig. 5 and Fig. 6).

\section{Discussion}

Several recent publications have identified adverse effects of HRT in a diverse group of disease processes, including stroke, ${ }^{12}$ breast cancer, ${ }^{13,14}$ osteoporosis $^{8}$ and urinary incontinence. ${ }^{7}$ Further, the Women's Health Initiative (WHI) trial, evaluating the effects of postmenopausal estrogen plus progesterone therapy was prematurely halted secondary to multiple reported adverse trends in the treatment arm. ${ }^{12-14}$ The results of our experiment align with these aforementioned studies, indicat- ing that extended term estrogen therapy (> $5 \mathrm{wk}$ ) worsened voiding dysfunction in our postpartum intravaginal balloon-dilated, ovariectomized rat model.

Using this model, we previously reported that birth trauma simulated by weighted ballooning and ovariectomy appears to contribute to SUI. ${ }^{9,11}$ We have also demonstrated that these rats rarely have UI during pregnancy or after ovariectomy alone without vaginal distension. Although the scenario does not create a true replica of prolonged labour as seen in humans, we believe this model is suitable for studying the urinary continence mechanism in the rat.

In our study, cystometric curve and the observation of urine (saline) leakage pattern clearly iden-

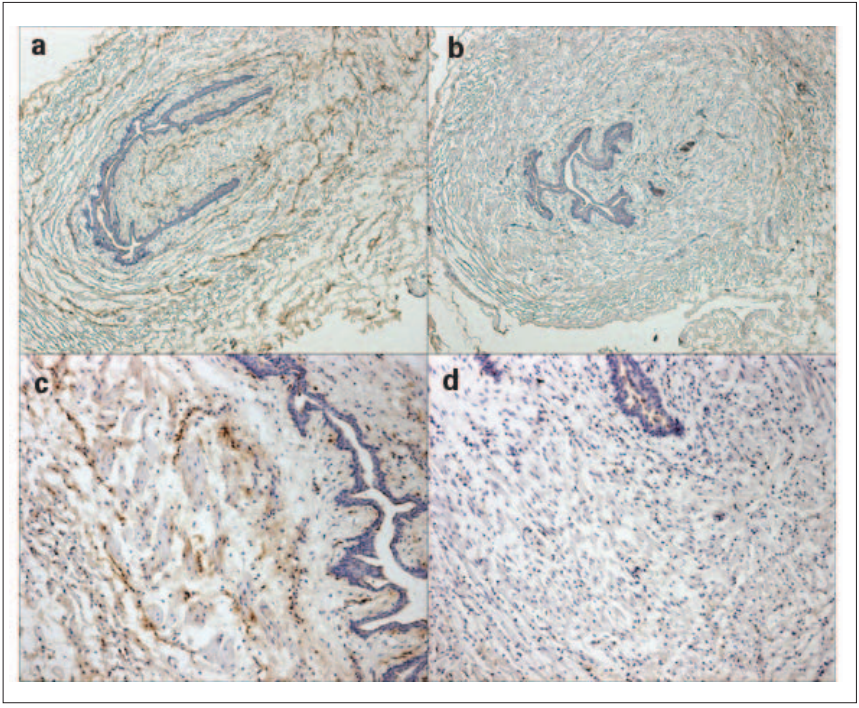

Fig. 3. Representative $\alpha_{1 \mathrm{~A}}$-adrenoceptor staining of cross sections of midurethra for placebo-treated (a and $c$ ) and estrogen-treated groups ( $b$ and d). There is a significant decrease of $\alpha_{1 A}$ (brown) staining in the estrogentreated group (original magnification a and b 40x, c and d 100x).

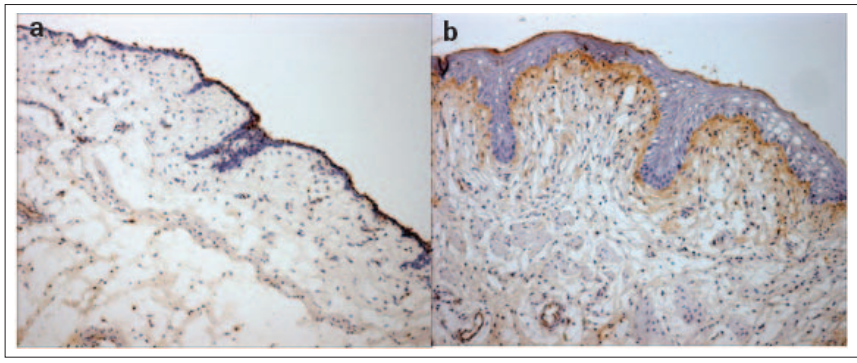

Fig. 4. Representative $\alpha_{1 A}$-adrenoceptor staining of vaginal tissue for placebotreated (a) and estrogen-treated groups (b). There is a much thicker epithelium and subepithelial layer, more muscle in the muscularis layer and increased $\alpha_{1 \mathrm{~A}}$ (brown) staining in the estrogen treatment group (original agnification 100x). 
Hayashi et al

tified rats with normal voiding patterns and those with abnormal leakage. Since the cystometry was performed under ketamine sedation, we could not determine whether the AV pattern was due to pure sphincter insufficiency or mixed with overactive bladder, we included all rats with abnormal voiding in the AV group. Future studies with conscious cystometry will be required to further study the AV patterns.

Using immunohistochemistry, we observed a significant downregulation of $\alpha_{1 A^{-}}$and $\alpha_{D}$-adrenoceptor expression in the endothelium and subendothelial regions of the urethral submucosa in rats treated with estrogen. Conversely, there was an increase in $\alpha_{1 A^{-}}$and $\alpha_{D^{-}}$-receptor

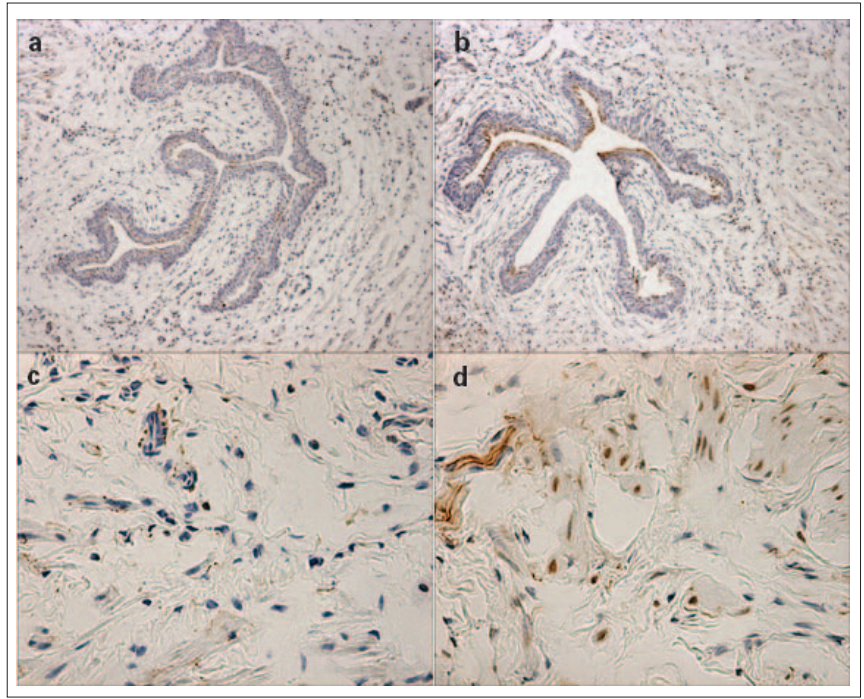

Fig. 5. Representative neuronal nitric oxide synthase (nNOS) staining of midurethral cross-sections for placebo-treated (a and c) and estrogentreated groups ( $b$ and $d)$. There is a significant increase of smooth muscle and epithelial nNOS (brown) staining in the estrogen-treated group (original magnification a and $b 40 x, c$ and $d 400 x)$.

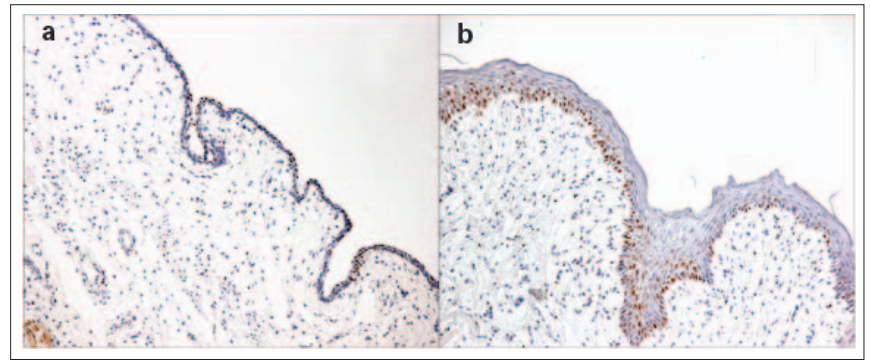

Fig. 6. Representative neuronal nitric oxide synthase (nNOS) staining of the vaginal epithelium for placebo-treated (a) and estrogen-treated groups (b). There is a significant increase of nNOS (brown) staining in the basal layer of epithelium of the estrogen-treatment group (original magnification 100x). immunostaining in vaginal tissues. This differential regulation of adrenoceptor in the urethra and vagina is intriguing and warrants further investigation (Table 3).

Urinary continence is believed to be maintained by sympathetic impulses through $\alpha_{1}$-adrenoceptors in the bladder neck and urethral smooth muscle..$^{15,16}$ Binding and molecular biological studies established the $\alpha_{1 \mathrm{~A}}$-adrenoceptor to be the predominant subtype in the urethra, responsible for the contractile responses of the urethra via elevated intracellular calcium levels to adrenergic agonists. ${ }^{17-19}$ Taki and colleagues ${ }^{20}$ demonstrated that $\alpha_{1}$-adrenoceptors play an important role in maintaining the resting urethral tonus of the female urethra. They divided the urethra into segments and administered noradrenaline (NA), an $\alpha_{1}$ agonist, demonstrating a concentration-dependent contraction with a peak amplitude in the middle to proximal urethra, while acetylcholine produced contractions only in the proximal urethra and bladder neck. Applying these results to our experiment, a downregulation of $\alpha_{1}$-adrenoceptors would contribute to a significant decrease in contraction and tone of urethral smooth muscle.

The 4 reported $\alpha_{1}$-adrenoceptor subtypes include $\alpha_{1 \mathrm{~A}}, \alpha_{1 B}, \alpha_{1 \mathrm{D}}$ and $\alpha_{1 L}{ }^{21}$ In the proximal female urethra, the expression of $\alpha_{1}$-adrenoceptor subtype mRNA has a reported ratio of 90:0:10 for $\alpha_{1 A}, \alpha_{1 B}$ and $\alpha_{1 D}$, respectively. ${ }^{22}$ However, Nishimatsu and colleagues ${ }^{23}$ reported that phenylephrine, a nonselective $\alpha_{1}$-adrenoceptor agonist, induced contractions through the $\alpha_{1 L}$ receptor, and not through the $\alpha_{1 \mathrm{~A}}$ subtype. The role of each of these $\alpha_{1}$-adrenoceptor subtypes in relation to urethral contractile function has yet to be delineated. Regardless, our results suggest that downregulation of both $\alpha_{1 \mathrm{~A}}$ and $\alpha_{1 \mathrm{D}}$ adrenoceptors may represent one mechanism by which impaired contraction of the urethra can lead to incontinence.

$\mathrm{NO}$, a neurotransmitter that causes relaxation of urethral smooth muscle, is produced by NOS, an enzyme located at several sites, including neurons. ${ }^{24,25}$ Recent studies suggest that adrenergic and NO-mediated nerves may function in a complex interactive manner and not as independent agents. ${ }^{17}$ In the rabbit urethra, released NO suppresses NA release from adrenergic nerve terminals; the reverse has also been reported. ${ }^{26}$ Perhaps our finding of upregulated urethral nNOS in the estrogen group results in smooth muscle relaxation 
by both local effect and $\alpha_{1}$-adrenoceptor downregulation with a net decrease in NA release.

Further investigation is needed to determine the intricate mechanisms causing SUI. Both molecular study and physiological animal experiments are needed to advance our knowledge and improve clinical management. Identifying the mechanism by which estrogen modulates the $\alpha_{1}$ adrenoceptor will be valuable not only to incontinence research but also to a broad spectrum of diseases.

\section{Conclusion}

Extended term estrogen therapy in a rat model of simulated birth trauma and ovariectomy resulted in a higher rate of incontinence. Immunohistochemical examination shows significant downregulation of urethral $\alpha_{1 \mathrm{~A}}$ and $\alpha_{1 \mathrm{D}}$ adrenoceptors and upregulation of nNOS in the urethra of estrogen-treated groups. These studies question the use of HRT in the treatment of postmenopausal incontinence.

From the Knuppe Molecular Urology Lab, Department of Urology, University of California, San Francisco, Calif.

Acknowledgements: This work is supported by NIDDK/NIH grants: R01 DK06965501 and P50 DK64538. Dr. A.J. Bella is the American Urological Association Foundation Robert J. Krane Scholar and a Royal College of Physicians and Surgeons (Canada) Detweiler Travelling Fellow.

This article has been peer reviewed.

Competing interests: None declared.

\section{References}

1. Anger JT, Saigal CS, Litwin MS; Urologic Diseases of America Project. The prevalence of urinary incontinence among community dwelling adult women: results from the National Health and Nutrition Examination Survey. J Urol 2006;175:601-4.

2. Cheater $F M$, Castleden CM. Epidemiology and classification of urinary incontinence. Baillieres Best Pract Res Clin Obstet Gynaecol 2000;14:183-205.

3. Robinson D, Cardozo L. The menopause and HRT. Urogenital effects of hormone therapy. Best Pract Res Clin Endocrinol Metab 2003;17:91-104.

4. Judge TG. The use of quinestradol in elderly incontinent women, a preliminary report. Gerontol Clin (Basel) 1969;11:159-64.

5. Siegel I, Zelinger BB, Kanter AE. Estrogen therapy for urogenital conditions in the aged. Am J Obstet Gynecol 1962;84:505-7.

6. Faber P, Heidenreich J. Treatment of stress incontinence with estrogen in postmenopausal women. Urol Int 1977;32:221-3.

7. Grady D, Brown IS, Vittinghoff E, et al. Postmenopausal hormones and incontinence: the Heart and Estrogen/Progestin Replacement Study. Obstet Gynecol 2001;97:116-20.

8. Goldstein SR, Nanavati N. Adverse events that are associated with the selective estrogen receptor modulator levormeloxifene in an aborted phase III osteoporosis treatment study. Am J Obstet Gynecol 2002;187:521-7.

9. Lin AS, Carrier $S$, Morgan DM, et al. Effect of simulated birth trauma on the urinary continence mechanism in the rat. Urology 1998;52:143-51.

10. Resplande J, Gholami SS, Graziottin TM, et al. Long-term effect of ovariectomy and simulated birth trauma on the lower urinary tract of female rats. J Urol 2002;168:323-30.

11. Sievert KD, Bakircioglu ME, Tsai T, et al. The effect of labor and/or ovariectomy on rodent continence mechanism - The neuronal changes. World J Urol 2004;22:244-50.

12. Wassertheil-Smoller $S$, Hendrix SL, Limacher $M$, et al. Effect of estrogen plus progestin on stroke in postmenopausal women: the Women's Health Initiative: a randomized trial. JAMA 2003;289:2673-84.

13. Gass M. Impact of WHI conclusions and ACOG guidelines on clinical practice. Int J Fertil Womens Med 2003;48:106-10.

14. Chlebowski RT, Hendrix SL, Langer RD, et al. Influence of estrogen plus progestin on breast cancer and mammography in healthy postmenopausal women: the Women's Health Initiative Randomized Trial. JAMA 2003;289:3243-53.

15. Anderson KE. Pharmacology of lower urinary tract smooth muscles and penile erectile tissues. Pharmacol Rev 1993;45:253-308.

16. Thien T, Delaere KP, Debruyne FM, et al. Urinary incontinence caused by prazosin. BMJ 1978;1:622-3.

17. Hatano A, Takahashi H, Tamaki M, et al. Pharmacological evidence of distinct alpha 1-adrenoceptor subtypes mediating the contraction of human prostatic urethra and peripheral artery. Br J Pharmacol 1994;113:723-8.

18. Testa R, Guarneri L, Ibba M, et al. Characterization of alpha 1-adrenoceptor subtypes in prostate and prostatic urethra of rat, rabbit, dog and man. Eur J Pharmacol 1993; 249:307-15.

19. Takahashi H, Takeda M, Shimura H, et al. Alpha-l adrenoceptor subtypes in the human female urethra-RT-PCR, in situ hybridization, and quantitative autoradiography. Neurourol Urodyn 1999;15:342-3.

20. Taki N, Taniguchi T, Okada K, et al. Evidence for predominant mediation of alpha Iadrenoceptor in the tonus of entire urethra of women. J Urol 1999;162:1829-32.

21. Graham RM, Perez DM, Hwa J, et al. Alpha 1-adrenergic receptor subtypes. Molecular structure, function, and signaling. Circ Res 1996;78:737-49.

22. Nasu K, Moriyama N, Fukasawa R, et al. Quantification and distribution of alpha ladrenoceptor subtype mRNAs in human proximal urethra. Br J Pharmacol 1998;123:1289-93.

23. Nishimatsu H, Moriyama N, Hamada K, et al. Contractile responses to alphal-adrenoceptor agonists in isolated human male and female urethra. BJU Int 1999;84:515-20.

24. Persson $K, A l m P$, Uvelius B, et al. Nitrergic and cholinergic innervation of the rat lower urinary tract after pelvic ganglionectomy. Am J Physiol 1998;274:R389-97.

25. Waldeck K, Ny L, Persson K, et al. Mediators and mechanisms of relaxation in rabbit urethral smooth muscle. Br J Pharmacol 1998;123:617-24.

26. Yoshida M, Inadome A, Murakami S. Pharmacological analysis of neurotransmitters contributing to lower urinary tract function [in Japanese]. Nippon Yakurigaku Zasshi 2003;121:307-16.

Correspondence: Dr. Tom F. Lue, Department of Urology, Knuppe Molecular Urology Laboratory, University of California, 400 Parnassus Ave., Box 0738, San Francisco CA 94143-0738; fax 415 476-8849; tlue@urology.ucs.edu 